\title{
Effect of adhesive strategy on non-carious cervical lesions pre-conditioned with bioactive glass air- abrasion: a randomised controlled clinical trial
}

Hussam Milly ( $\nabla$ milly.hussam@gmail.com )

Damascus University https://orcid.org/0000-0003-1852-0939

Humam Karkoutly

Damascus University

Avijit Banerjee

King's College London Dental Institute

\section{Research article}

Keywords: Non-carious cervical lesions (NCCLs), air-abrasion, bio-active glass (BAG), resin composite, etch and rinse adhesive, self-etch adhesive

Posted Date: February 17th, 2021

DOl: https://doi.org/10.21203/rs.3.rs-238068/v1

License: (c) (i) This work is licensed under a Creative Commons Attribution 4.0 International License. Read Full License 


\section{Abstract}

Background: This double-blind randomised controlled clinical trial evaluated the effect of adhesive strategy on the performance of resin composite restorations in non-carious cervical lesions (NCCLs) preconditioned using bioactive glass air-abrasion.

Methods: One hundred teeth with NCCLs were assigned randomly into four experimental groups $(n=25)$; A: air-abrasion + etch \& rinse adhesive (AA+ER), B: air-abrasion + self-etch adhesive (AA+SE), C: etch \& rinse adhesive (ER) and D: self-etch adhesive (SE). NCCLs were restored clinically using conventional resin composite placed incrementally. The lesions and restorations were evaluated at baseline, 3-months and 6-months according to the World Dental Federation (FDI) criteria. The level of statistical significance was established at $p=0.05$.

Results: An increased retention rate was observed at 6-month recall within the AA+ER group. The retention rate of NCCLs restoration was affected significantly by the height of the NCCLs. NCCLs width and the height affected significantly the marginal adaptation of the restorations.

Conclusion: Etch-and-rinse adhesive revealed a statistical improved retention rate when compared to selfetch adhesive in NCCLs pre-conditioned with bioactive glass air-abrasion at 6-months recall. NCCLs' physical characteristics affected significantly the resin composite restoration performance.

This clinical trial was registered in clinicaltrial.gov clinical registry under protocol \# NCT04578717.

\section{Background}

The restoration of non-carious cervical lesions (NCCLs) exhibits a clinical challenge attributed to the lack of a macro-mechanical cavity retention, the location of the cervical margins close to the gingivae, the cervical flexure that occurs in the tooth during function and the existence of hyper-mineralised dentine surfaces [1]. Static / cyclic stress and erosion / abrasion alter significantly the dentine structure and morphology which in turn, reduces the survival rate of resin composite restorations [2, 3]. Previous investigations have revealed that the retention rate and marginal discoloration of resin composite restorations in NCCLs are not significantly influenced by resin type or viscosity [4-6]. Resin composite restorations placed with etch-and-rinse (ER), self-etch (SE) or universal adhesive systems produced similar retention and post-operative sensitivity (POS), implying that the adhesive strategy does not influence their clinical behaviour in NCCLs $[2,7,8]$. Dentine surface roughening may enhance the retention rate of resin composite restorations in NCCLs since this disrupts the hypermineralised dentine and improves hybrid layer formation [9]. However, it has been demonstrated that dentine roughening with a diamond bur for $5 \mathrm{sec}$ in a rotary air-turbine handpiece with water cooling did not significantly affect the clinical behaviour of resin composite restorations placed in NCCLs [10].

Treating dental hard tissues with air-abrasion technology produces rounded cavo-surface and internal line angles, a halo of abraded enamel and a microscopic surface roughness [11]. These features are 
optimal for the adhesion of contemporary dental materials. Bio-active glass $45 \mathrm{~S} 5$ (BAG) has been introduced as an abrasive powder in air-abrasion to benefit from its unique characteristics including remineralisation and its ability to remove selectively restorations/damaged tooth structure [12]. Preconditioning enamel white spot lesions using BAG air-abrasion removed an ultra-thin surface layer, increased the surface area and enhanced its remineralisation potential [13]. BAG air-abrasion had a significant longer term desensitising effect when used for professional dental stain removal [14]. A bioactive smear layer was detected using confocal laser microscopy (CLM) and atomic force miscopy (AFM) when dentine was treated with BAG air-abrasion [15]. This therapeutic approach permits the incorporation of BAG particles within SE adhesives protecting the bond interface and enhancing remineralisation [16].

Evaluating the clinical effectiveness of adhesive strategy in NCCLs pre-conditioned using BAG airabrasion has not been reported in the dental literature. Therefore, the objective of this double-blind, randomised controlled clinical trial was to assess the effect of ER and SE adhesive systems on the performance of resin composite restorations in NCCLs pre-conditioned with BAG air-abrasion. The null hypothesis investigated in this study was that ER and SE adhesives presented similar clinical performance in the NCCLs pre-conditioned with BAG air-abrasion over the course of 6 months.

\section{Methods}

\section{Study Design}

This double-blind, randomised clinical trial was carried out in accordance with CONSORT guidelines [17]. This study was reviewed by the Health Research Ethics Board at Faculty of Dental Medicine (N34792018), and performed in the clinics of Restorative Dentistry department, from October 2018 to February 2020.

\section{Protocol registration}

This trial is registered at clinicaltrial.gov (NCT04578717). Written consent was obtained from all participants.

\section{Sample size calculation}

The sample size was calculated using G-Power 3.1 software. Twenty five teeth were recruited per group to detect a significance level at $5 \%$ with an $80 \%$ power. Means and standard deviations of the failure rate were set at (5.8 \pm 4.9$)$ in ER groups and $(4.4 \pm 4.6)$ in SE groups in accordance with a previous study [3].

\section{Inclusion and exclusion criteria}

The inclusion criteria for patients recruited to this study were that they needed to be in good general and oral health, be at least 19 years old, and be available for follow-up visits. The exclusion criteria were a chronic therapeutic drug history, rampant uncontrolled caries, periodontal disease, pregnancy or lactation, orthodontic appliance use, evidence of xerostomia, evidence of TMD, and known sensitivity to resin acrylates. With respect to the NCCLs, the inclusion criteria were they needed to present at least $1 \mathrm{~mm}$ 
deep, involving enamel and dentine of vital teeth. Enamel margins should be present at the coronal aspect of the lesion. NCCL exclusion criteria were teeth with periapical pathology, non-vital or previous root canal therapy, symptoms of pulp pathology, previous pulp capping $[7,10]$.

\section{Randomisation and sample allocation}

One hundred teeth with NCCLs meeting the inclusion criteria were assigned randomly into four experimental groups $(n=25) ; A$ : BAG air-abrasion + ER, B: BAG air-abrasion + SE, C: ER and D: SE. The randomisation was conducted on an intra-oral basis. This procedure was fulfilled by three sequential steps, allocation teeth with NCCLs, allocation of BAG air-abrasion preconditioning and finally allocation of the adhesive system to be used.

\section{Intervention}

The parameters of NCCLs were characterised prior to the clinical intervention using open-software platform, ImageJ (ImageJ, MD, USA). These parameters included the width/depth/height of the cavity and the lesion geometry $\left(>45^{\circ}, 45^{\circ}-90^{\circ}, 90^{\circ}-135^{\circ}\right.$, and $\left.<135^{\circ}\right)$. Dentine hypermineralisation was measured according to a clinical dentine sclerosis scale [18]. Additionally, the presence of attrition facets and spontaneous sensitivity were recorded. Gingival retraction cord number 00 (SURE-Cord, Sure-endo Sure Dent Co., Korea) was inserted into the gingival sulcus and the teeth were isolated with a rubber dam. No preparation or bevelling of margins were conducted to the NCCLs in all experimental groups.

In the BAG air-abrasion groups, a clinical air-abrasion unit (KaVo Dental, Biberach, Germany) was used to pre-condition the NCCLs with bio-active glass 45S5 (ProSylc-CR, Velopex, Harlesden, UK) powder for 10 $\mathrm{sec}$. The operating parameters were; powder particle size, $20 \mu \mathrm{m}$; nozzle-lesion distance, $5 \mathrm{~mm}$; nozzle angle, $90 \circ$; the internal nozzle diameter, $900 \mu \mathrm{m}$ and air pressure, $20 \mathrm{psi}$. The powder reservoir was refilled to a pre-determined line consistently to standardise the emitted powder flow rate [13].

For the ER groups, enamel and dentine were etched using $37 \%$ orthophosphoric acid (N-Etch, Ivoclar Vivadent, Schaan, Liechtenstein) for $30 \mathrm{sec}$ and $15 \mathrm{sec}$ respectively. NCCLs were rinsed thoroughly for 20 sec to remove the acid. Excess water was removed using a cotton pellet to leave a moist dentine surface. One coat of adhesive (Adper ${ }^{\mathrm{TM}}$ Single Bond 2, 3M, USA) was gently agitated on the entire enamel and dentine surface for $20 \mathrm{sec}$ using a sterile microbrush, air-dried for $10 \mathrm{sec}$ and light-cured for $20 \mathrm{sec}$ using LED curing light (Woodpecker, output $>800 \mathrm{~mW} / \mathrm{cm}^{2}$ ). The light intensity of curing unit was calibrated daily using curing radiometer (Demetron 100, SDS-Kerr; Orange, CA, USA). In SE groups, the adhesive (Adper $^{\text {TM }}$ Easy Bond, 3M Oral Care, Neuss, Germany) was applied to enamel and dentine for $20 \mathrm{sec}$, gently air-dried for $10 \mathrm{sec}$ and light cured for $20 \mathrm{sec}$.

NCCLs were restored using Filtek ${ }^{\mathrm{TM}} \mathrm{Z} 250 \mathrm{XT}$ (3M ESPE, USA) resin composite, placed in $2 \mathrm{~mm}$ increments and light-cured for $20 \mathrm{~s}$ per increment. Final contour was achieved with a fine diamond rotary instrument (Heico, Steinach, Switzerland) followed by finishing and polishing using Optrapol polishing kit (Ivoclar Vivadent, Schaan, Liechtenstein).

\section{Clinical evaluation}


Clinical evaluation was performed by two independent, trained examiners. The examiners and patients were blinded to the group assignment. Each restoration was evaluated by the same examiners at baseline (one week after restoration placement), 3 months and 6 months. The restoration evaluation was conducted according to World Federation criteria (FDI) [19].

\section{Statistical analysis}

Data was analysed using IBM SPSS 23. Descriptive statistics were used to report the frequency distribution of the evaluated criteria. Differences in the ratings of the experimental groups at baseline and after 3 and 6 months were evaluated using the Friedman Chi-square test at $p=0.05$.

\section{Results}

Two trained dentists evaluated 162 teeth initially (Fig. 1). Fifteen participants with 100 NCCLs meeting the inclusion criteria were enrolled in this study. Baseline details relative to the participants and the NCCLs characteristics are presented in Table 1. Two participants with four NCCLs did not attend the 6month follow-up as they had relocated. The number of evaluated restorations for each group according to FDI criteria is shown in Table 2. 
Table 1

Distribution of NCCLs according to the studied patients and the characteristics of lesions

\begin{tabular}{|c|c|c|}
\hline Characteristics of research subjects & No. of lesions/subjects & $\%$ \\
\hline \multicolumn{3}{|l|}{ Gender distribution } \\
\hline Male & 8 & 53.3 \\
\hline Female & 7 & 46.7 \\
\hline \multicolumn{3}{|l|}{ Age distribution, $y$} \\
\hline $18-29$ & 1 & 6.7 \\
\hline $30-39$ & 2 & 13.3 \\
\hline $40-49$ & 1 & 6.7 \\
\hline$<49$ & 11 & 73.3 \\
\hline \multicolumn{3}{|l|}{ Characteristics of lesions } \\
\hline \multicolumn{3}{|l|}{ Shape, ${ }^{\circ}$ of angle } \\
\hline$<45$ & 2 & 2.0 \\
\hline $45-90$ & 44 & 44.0 \\
\hline $90-135$ & 28 & 28.0 \\
\hline$>135$ & 26 & 26.0 \\
\hline \multicolumn{3}{|l|}{ Cervico-incisal height ( $\mathrm{mm})$} \\
\hline$\neg<1.5$ & 4 & 4.0 \\
\hline $1.5-2.5$ & 42 & 42.0 \\
\hline $2.5-4.0$ & 29 & 29.0 \\
\hline$>4.0$ & 25 & 25.0 \\
\hline \multicolumn{3}{|l|}{ Width $(\mathrm{mm})$} \\
\hline$<4.0$ & 45 & 45.0 \\
\hline $4.0-5.9$ & 48 & 48.0 \\
\hline $6.0-7.9$ & 7 & 7.0 \\
\hline$>8.0$ & 0 & 0.0 \\
\hline \multicolumn{3}{|l|}{ Depth $(\mathrm{mm})$} \\
\hline Flat (1.0-1.5) & 29 & 29.0 \\
\hline
\end{tabular}




\begin{tabular}{|lll|}
\hline Characteristics of research subjects & No. of lesions/subjects & $\%$ \\
\hline Medium (1.5-2.5) & 47 & 47.0 \\
\hline Deep (>2.5) & 24 & 24.0 \\
\hline Degree of sclerotic dentin & & \\
\hline 1 & 14 & 14.0 \\
2 & 53 & 53.0 \\
3 & 30 & 30.0 \\
4 & 3 & 3.0 \\
\hline Attrition facets & & \\
\hline Yes & 67 & 67.0 \\
\hline No & 33 & 33.0 \\
\hline
\end{tabular}


Table 2

Number of restorations in each experimental group classified according to the FDI Criteria Criteria* Baseline (after 1 week) After 3 months After 6 months

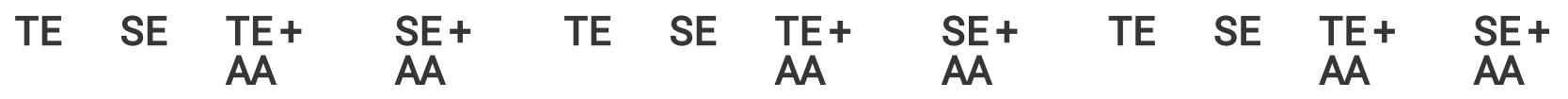

1. Marginal staining

$\begin{array}{lllllllllllll}\text { VG } & 25 & 25 & 25 & 25 & 17 & 15 & 20 & 16 & 15 & 13 & 18 & 13 \\ \text { GO } & - & - & - & - & 1 & 1 & - & - & - & 2 & - & - \\ \text { SS } & - & - & - & - & - & - & - & - & 1 & - & 1 & - \\ \text { UN } & - & - & - & - & - & - & - & - & - & - & - & - \\ \text { PO } & - & - & - & - & - & - & - & - & - & - & - & - \\ \text { 2. Fractures and retention } & & & & & & & & \\ \text { VG } & 25 & 25 & 25 & 25 & 18 & 16 & 20 & 16 & 15 & 13 & 19 & 12 \\ \text { GO } & - & - & - & - & - & - & - & - & - & 2 & - & 1 \\ \text { SS } & - & - & - & - & - & - & - & - & 1 & - & - & - \\ \text { UN } & - & - & - & - & - & - & - & - & - & - & - & - \\ \text { PO } & - & - & - & - & 7 & 9 & 5 & 9 & 2 & 1 & 1 & 3\end{array}$

3. Marginal adaptation

$\begin{array}{lllllllllllll}\text { VG } & 22 & 23 & 24 & 23 & 13 & 12 & 17 & 11 & 8 & 9 & 14 & 8 \\ \text { GO } & 3 & 2 & 1 & 2 & 5 & 4 & 3 & 5 & 7 & 6 & 4 & 5 \\ \text { SS } & - & - & - & - & - & - & - & - & 1 & - & 1 & - \\ \text { UN } & - & - & - & - & - & - & - & - & - & - & - & - \\ \text { PO } & - & - & - & - & - & - & - & - & - & - & - & -\end{array}$

4. Post-operative sensitivity

$\begin{array}{lllllllllllll}\text { VG } & 22 & 22 & 24 & 24 & 15 & 15 & 17 & 13 & 12 & 13 & 15 & 10 \\ \text { GO } & 2 & 3 & 1 & 1 & 2 & 1 & 3 & 3 & 3 & 2 & 4 & 3 \\ \text { SS } & 1 & - & - & - & 1 & - & - & - & 1 & - & - & - \\ \text { UN } & - & - & - & - & - & - & - & - & - & - & - & - \\ \text { PO } & - & - & - & - & - & - & - & - & - & - & - & -\end{array}$

5. Secondary caries 


\begin{tabular}{|c|c|c|c|c|c|c|c|c|c|c|c|c|}
\hline \multirow[t]{2}{*}{ Criteria* } & \multicolumn{4}{|c|}{ Baseline (after 1 week) } & \multicolumn{4}{|c|}{ After 3 months } & \multicolumn{4}{|c|}{ After 6 months } \\
\hline & TE & SE & $\begin{array}{l}\mathrm{TE}+ \\
\mathrm{AA}\end{array}$ & $\begin{array}{l}S E+ \\
A A\end{array}$ & TE & SE & $\begin{array}{l}\text { TE + } \\
\text { AA }\end{array}$ & $\begin{array}{l}S E+ \\
A A\end{array}$ & TE & SE & $\begin{array}{l}\text { TE + } \\
\text { AA }\end{array}$ & $\begin{array}{l}\text { SE + } \\
\text { AA }\end{array}$ \\
\hline VG & 25 & 25 & 25 & 25 & 18 & 16 & 20 & 16 & 16 & 15 & 19 & 13 \\
\hline GO & - & - & - & - & - & - & - & - & - & - & - & - \\
\hline SS & - & - & - & - & - & - & - & - & - & - & - & - \\
\hline UN & - & - & - & - & - & - & - & - & - & - & - & - \\
\hline PO & - & - & - & - & - & - & - & - & - & - & - & - \\
\hline $\begin{array}{l}\text { Abbrevia } \\
\text { * VG: clin } \\
\text { unsatisfa }\end{array}$ & ons: & $E:$ tot & $\begin{array}{l}\text { I-etchir } \\
\text { od; GO: } \\
\mathrm{or}\end{array}$ & $\begin{array}{l}\text { dhesiv } \\
\text { nically }\end{array}$ & roup & SE: $S$ & $\begin{array}{l}\text { f-etchi } \\
\text { ally su }\end{array}$ & $\begin{array}{l}\text { adhesi } \\
\text { sient/s }\end{array}$ & $\begin{array}{l}\text { group } \\
\text { facto }\end{array}$ & AA: & ir-abras & \\
\hline
\end{tabular}

NCCL restorations retention rates at 3-month recall were $72 \%$ for $E R, 64 \%$ for $S E, 80 \%$ for $A A+E R$, and $64 \%$ for AA + SE with no statistical differences between the four experimental groups $(p>0.05)$. An improved retention rate was observed at 6-month recall within the ER $+A A$ group (67\%), with no statistical differences when compared to ER $(64 \%)$ and SE $(60 \%)$ groups $(p>0.05)$. However, the retention rate in ER + AA was significantly better than that of SE + AA $(52 \%)$ groups at 6-month recall $(p<0.05)$. The retention rate of NCCL restorations were affected significantly $(p=0.04)$ by the height of NCCLs. Seventy three per cent of lost restorations at 6-month recall were placed in lesions that surpassed $2.5 \mathrm{~mm}$ in height.

Minor discrepancies in marginal adaptation were detected within 17 and 24 restorations at 3-month and 6-month recalls respectively. No statistical differences in the marginal adaptation were recorded between the four experimental groups at 3-month and 6-month recalls $(p>0.05)$. NCCLs width and the height affected significantly the marginal adaptation of the restorations ( $p=0.038 \& p=0.035$ respectively) at 6month recall. The percentages of adaptation defects were $40.9 \%$ in the NCCLs with $0.4-5.9 \mathrm{~mm}$ in width and $58.3 \%$ in NCCLs with $1.5-2.5 \mathrm{~mm}$ in height. The presence of occlusal attrition facets in the teeth increased significantly $(p=0.043)$ the percentage of the adaptation defects $(75 \%)$ at 6-month recall. Seventy one percent of the adaptation defects was observed in NCCLs with dentine hypermineralisation at 6-month recall $(p=0.007)$.

Ten restorations at 3-month recall and 13 restorations at 6-month recall showed mild post-operative sensitivity. The four experimental groups revealed similar post-operative sensitivity at 3- and 6- month recall $(p>0.05)$. Dentine hypermineralisation reduced significantly the post-operative sensitivity at 6 month recall $(p=0.002)$. Twenty three percent of NCCLs restorations with no dentine sclerosis reported postoperative sensitivity at 6 -month recall.

Minor discrepancies were observed in two restorations at 3-month recall and in four restorations at 6month recall. No statistical differences in marginal discoloration were detected between the four 
experimental groups at the 3 - and 6 - month recall $(p>0.05)$. No secondary caries was detected in any restoration placed in the present study.

\section{Discussion}

There is a wide variation in the operative management of NCCLs with a lack of randomised clinical trials that provide clinicians with a standard clinical protocol $[3,8]$. To the best of the authors' knowledge, this is the first clinical trial to evaluate the effect of adhesive strategy on NCCLs pre-conditioned with BAG airabrasion. The findings of the present clinical trial showed that the retention was not significantly enhanced following air-abrasion, in agreement with previous in-vitro investigations [20-23].

The current clinical trial advocates the use of ER rather than SE adhesive when the NCCLs is preconditioned using BAG air-abrasion as a significant increase in the retention rate of resin composite restorations was detected in $A A+E R$ group when compared to $A A+S E$ group. Treating dentine with $B A G$ air-abrasion sealed the orifices of dentine tubules, reduced the dentine permeability and stabilised the bond strength values $[15,16,24]$. BAG particles may help to remineralise carious dental tissues and demineralised dentine collagen fibres [24]. Dentine surface treated with air-abrasion is covered by an irregular, amorphous, thin smear layer $[20,25]$. The incorporation of self-adhesive agent with this irregular smear layer may explain the reduction of the retention rate observed in AA-SE group. The preparing method affects the thickness and uniformity of resin-smear complex which in turn, affects the interaction of self-etch adhesive [26].

On the other hand, the characteristics of dental surfaces pre-conditioned with air-abrasion may do not deteriorate the performance of ER adhesive. These features include surface micro-roughness, increased surface area and a featureless stippled surface appearance $[13,20]$. Although these findings are expected to improve the retention of resin composite restorations in clinical NCCLs, the findings of the present clinical trial showed that the retention was not significantly enhanced following air-abrasion with bioactive glass particles in ER group. The interaction of air-abrasion with the dentine surface might not be sufficient to alter the resin composite performance in vivo. Several biological factors may further influence the performance of such restorations including numerous microorganisms, pH fluctuating, masticatory cyclic loading and thermal cycling.

NCCL dimensional characteristics affected significantly the resin composite restoration performance. This clinical correlation needs further investigation to be used to improve assessment of prognosis and allow modifications to treatment protocols to be agreed. The height of NCCLs affected significantly the retention rate of resin composite restorations. Increasing the height in a lesion with divergent walls, reduces further the mechanical retention. In this study, increasing the width and the height of NCCLs affected significantly the marginal adaptation of the restorations. This increase leads to more vulnerable margins that can breakdown due to strains caused by eccentric occlusal forces, or acidic erosive or abrasive forces [27-29]. 
At 6-month recall, marginal adaptation had a significant relation with the occurrence of attrition facets. The continuous exposure to compressive/shear stresses leads to the attrition of dental tissues. Stresses accumulate in the cervical areas and consequently high levels of strain are formed, possibly affecting restorations retention and marginal integrity [30,31]. NCCLs restorations with no dentine hypermineralisation reported post-operative sensitivity at 6-month recall, whilst NCCLs restorations with hypermineralisation present had no significant change in sensitivity response. Hypermineralised dentine contains higher amounts of mineral crystal deposits that block the exposed dentine tubules, which in turn reduces the fluid movement in response to stimuli [32].

\section{Conclusion}

The null hypothesis investigated in this study is disproved as the retention rate of resin composite restorations in ER group was significantly better than that in SE group in pre-conditioned NCCLs at 6month recall. Pre-conditioning the dentine with bioactive glass air-abrasion did not improve the clinical performance of NCCLs restorations in both ER and SE adhesive groups at 6-month recall. NCCLs physical characteristics affected significantly resin composite restoration performance. Clinicians are advocated to be aware about the critical role of NCCLs' physical characteristics in the success of resin composite restorations.

\section{Abbreviations}

NCCLs: Non-carious cervical lesions; ER: etch-and-rinse; SE: self-etch; BAG: Bio-active glass 45S5; AA: airabrasion.

\section{Declarations}

\section{Acknowledgements}

The authors would like to thank all the patients who participated in the study.

\section{Authors' contributions}

$\mathrm{HM}$ and $\mathrm{AB}$ participated in designing the study, writing the manuscript and revising it before submission. $\mathrm{HK}$ and $\mathrm{HM}$ collected the data and did the field work. All authors read and approved the final manuscript.

\section{Funding}

The project was funded by Damascus University, Syria.

\section{Availability of data and materials}

The datasets used and/or analyzed during the present study are available from the corresponding author upon a reasonable request. 


\section{Ethics approval and consent to participate}

Ethical Approval was obtained from the ethics committee of the Faculty of Dentistry in Damascus University, Syria; (N3479-2018). A written informed consent was obtained from all participants.

\section{Consent for publication}

Not applicable.

\section{Competing interests}

The authors declare that they have no competing interests.

\section{References}

1. Grippo JO, Simring M, Coleman TA: Abfraction, abrasion, biocorrosion, and the enigma of noncarious cervical lesions: A 20-year perspective. J Esthet Restor Dent Dentistry 2012, 24(1):10-23.

2. Zanatta R, Silva T, Esper M, Bresciani E, Gonçalves S, Caneppele T: Bonding Performance of Simplified Adhesive Systems in Noncarious Cervical Lesions at 2-year Follow-up: A Double-blind Randomized Clinical Trial. Oper Den 2019.

3. Peumans M, De Munck J, Mine A, Van Meerbeek B: Clinical effectiveness of contemporary adhesives for the restoration of non-carious cervical lesions. A systematic review. Dent Mater 2014, 30(10):1089-1103.

4. Kubo S, Yokota H, Yokota H, Hayashi Y: Three-year clinical evaluation of a flowable and a hybrid resin composite in non-carious cervical lesions. . J Dent 2010, 38(3):191-200.

5. Karaman E, Yazici AR, Ozgunaltay G, Dayangac B: Clinical evaluation of a nanohybrid and a flowable resin composite in non-carious cervical lesions: 24-month results. J Adhes Dent 2012, 14(5).

6. Szesz A, Parreiras S, Martini E, Reis A, Loguercio A: Effect of flowable composites on the clinical performance of non-carious cervical lesions: A systematic review and meta-analysis. J Dent 2017, 65:11-21.

7. Lawson NC, Robles A, Fu CC, Lin CP, Sawlani K, Burgess JO: Two-year clinical trial of a universal adhesive in total-etch and self-etch mode in non-carious cervical lesions. J Dent 2015, 43(10):12291234.

8. Schroeder M, Correa IC, Bauer J, Loguercio AD, Reis A: Influence of adhesive strategy on clinical parameters in cervical restorations: A systematic review and meta-analysis. J Dent 2017, 62:36-53.

9. Mahn E, Rousson V, Heintze S: Meta-analysis of the influence of bonding parameters on the clinical outcome of tooth-colored cervical restorations. J Adhes Dent 2015, 17(5):391-403.

10. Loguercio AD, Luque-Martinez IV, Fuentes S, Reis A, Munoz MA: Effect of dentin roughness on the adhesive performance in non-carious cervical lesions: A double-blind randomized clinical trial. J Dent 2018, 69:60-69. 
11. Laurell KA, Hess JA: Scanning electron micrographic effects of air-abrasion cavity preparation on human enamel and dentin. Quintessence Int 1995, 26(2).

12. Milly H, Festy F, Watson TF, Thompson I, Banerjee A: Enamel white spot lesions can remineralise using bio-active glass and polyacrylic acid-modified bio-active glass powders. J Dent 2014, 42(2):158-166.

13. Milly H, Festy F, Andiappan M, Watson TF, Thompson I, Banerjee A: Surface pre-conditioning with bioactive glass air-abrasion can enhance enamel white spot lesion remineralization. Dent Mater 2015, 31(5):522-533.

14. Banerjee A, Hajatdoost-Sani M, Farrell S, Thompson I: A clinical evaluation and comparison of bioactive glass and sodium bicarbonate air-polishing powders. J Dent 2010, 38(6):475-479.

15. Sauro S, Pashley DH: Strategies to stabilise dentine-bonded interfaces through remineralising operative approaches-State of The Art. Int J Adhes Adhes 2016, 69:39-57.

16. Sauro S, Watson TF, Thompson I, Banerjee A: One-bottle self-etching adhesives applied to dentine airabraded using bioactive glasses containing polyacrylic acid: an in vitro microtensile bond strength and confocal microscopy study. J Dent 2012, 40(11):896-905.

17. Schulz KF, Altman DG, Moher D: CONSORT 2010 Statement: updated guidelines for reporting parallel group randomised trials. BMC Med 2010, 8(1):18.

18. Swift Jr E, Perdigão J, Heymann H, Wilder Jr A, Bayne S, May Jr K, Sturdevant J, Roberson T: Eighteen-month clinical evaluation of a filled and unfilled dentin adhesive. J Dent 2001, 29(1):1-6.

19. Hickel R, Peschke A, Tyas M, Mjör I, Bayne S, Peters M, Hiller K-A, Randall R, Vanherle G, Heintze SD: FDI World Dental Federation: clinical criteria for the evaluation of direct and indirect restorationsupdate and clinical examples. Clin Oral Investi 2010, 14(4):349-366.

20. Freeman R, Varanasi S, Meyers IA, Symons AL: Effect of air abrasion and thermocycling on resin adaptation and shear bond strength to dentin for an etch-and-rinse and self-etch resin adhesive. Dent Mater J 2012:1203190242-1203190242.

21. Manhart J, Mehl A, Schroeter R, Obster B, Hickel R: Bond strength of composite to dentin treated by air abrasion. Oper Den 1999, 24(4):223.

22. Roeder L, Berry 3rd E, You C, Powers J: Bond strength of composite to air-abraded enamel and dentin. Oper Den 1995, 20(5):186-190.

23. Los S, Barkmeier WW: Effects of dentin air abrasion with aluminum oxide and hydroxyapatite on adhesive bond strength. Oper Den 1994, 19(5):169-175.

24. Sauro S, Osorio R, Watson TF, Toledano M: Therapeutic effects of novel resin bonding systems containing bioactive glasses on mineral-depleted areas within the bonded-dentine interface. J Mater Sci Mater Med 2012, 23(6):1521-1532.

25. Oliveira ACM, Lima LM, Pizzolitto AC, Santos-Pinto L: Evaluation of the smear layer and hybrid layer in noncarious and carious dentin prepared by air abrasion system and diamond tips. Microsc Res Tech 2010, 73(6):597-605. 
26. Mine A, De Munck J, Cardoso MV, Van Landuyt KL, Poitevin A, Van Ende A, Matsumoto M, Yoshida Y, Kuboki T, Yatani H: Dentin-smear remains at self-etch adhesive interface. Dent Mater 2014, 30(10):1147-1153.

27. Pereira FA, Zeola LF, de Almeida Milito G, Reis BR, Pereira RD, Soares PV: Restorative material and loading type influence on the biomechanical behavior of wedge shaped cervical lesions. Clin Oral Investi 2016, 20(3):433-441.

28. Michael J, Townsend G, Greenwood L, Kaidonis J: Abfraction: separating fact from fiction. Aust Dent J 2009, 54(1):2-8.

29. Grippo JO, Simring M, Schreiner S: Attrition, abrasion, corrosion and abfraction revisited: a new perspective on tooth surface lesions. J Am Dent Assoc 2004, 135(8):1109-1118.

30. Soares P, Machado A, Zeola L, Souza P, Galvão A, Montes T, Pereira A, Reis B, Coleman T, Grippo J: Loading and composite restoration assessment of various non-carious cervical lesions morphologies-3D finite element analysis. Aust Dent J 2015, 60(3):309-316.

31. Soares P, Souza L, Veríssimo C, Zeola L, Pereira A, Santos-Filho P, Fernandes-Neto A: Effect of root morphology on biomechanical behaviour of premolars associated with abfraction lesions and different loading types. J Oral Rehabil 2014, 41(2):108-114.

32. van Dijken JW: A prospective 8-year evaluation of a mild two-step self-etching adhesive and a heavily filled two-step etch-and-rinse system in non-carious cervical lesions. Dent Mater 2010, 26(9):940-946.

\section{Figures}




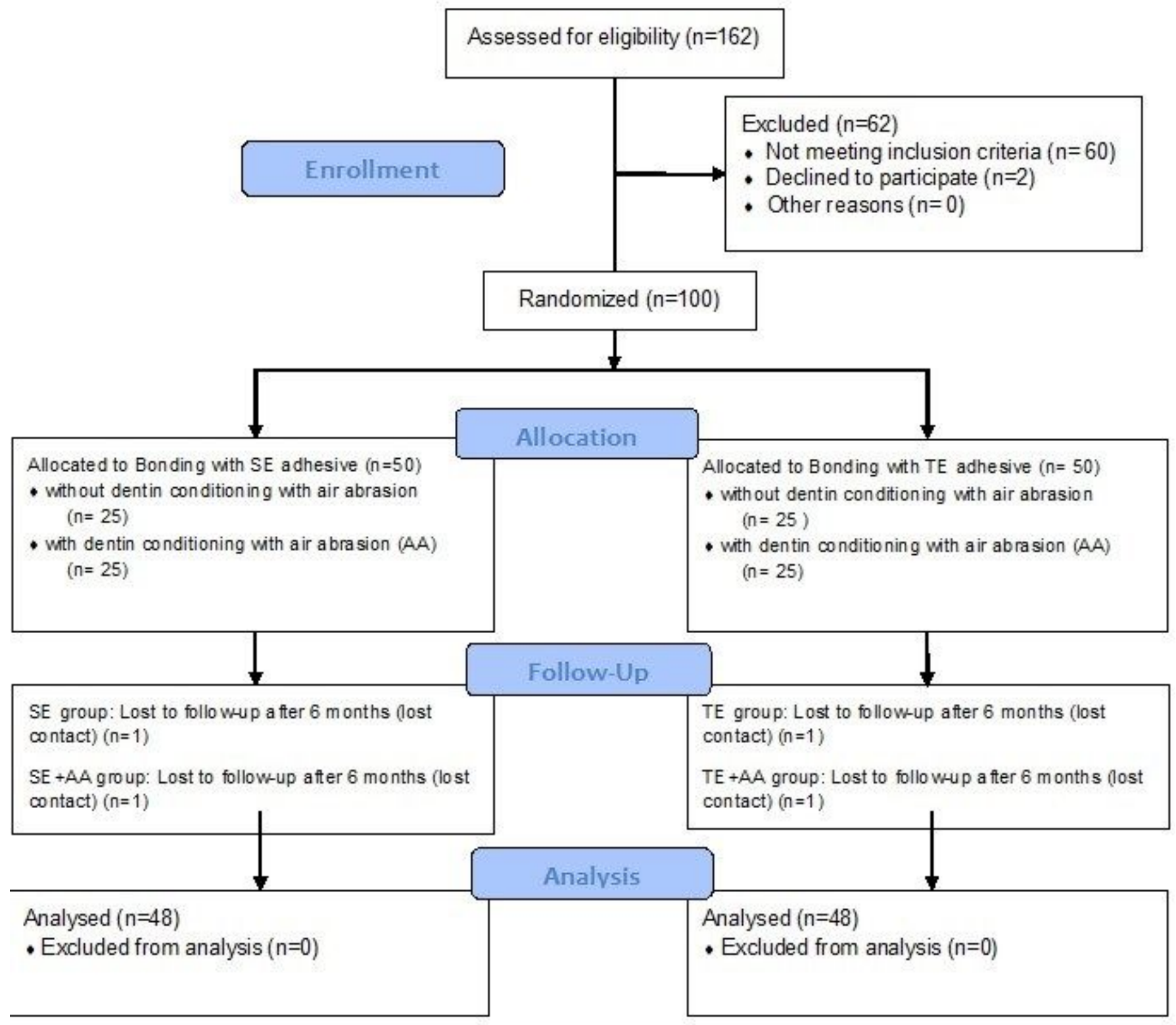

\section{Figure 1}

CONSORT flow diagram. TE: total-etching adhesive group, SE: self-etching adhesive group, AA: Airabrasion

\section{Supplementary Files}

This is a list of supplementary files associated with this preprint. Click to download. 
- CONSORT2010Checklist.doc

Page 16/16 